\title{
Genetic algorithms to optimize base station sitting in WCDMA networks
}

\author{
Najat Erradi \\ Laboratory Information Systems and Telecommunications \\ Faculty of Sciences. University Abdelmalek Essaadi \\ Tetouan 93000, Morocco.
}

Fadoua Thami Alami

Laboratory Information Systems and Telecommunications Faculty of Sciences. University Abdelmalek Essaadi

Tetouan 93000, Morocco.

\author{
Noura Aknin \\ Laboratory Information Systems and Telecommunications \\ Faculty of Sciences. University Abdelmalek Essaadi \\ Tetouan 93000, Morocco.
Ahmed El Moussaoui
Laboratory Information Systems and Telecommunications Faculty of Sciences. University Abdelmalek Essaadi Tetouan 93000, Morocco.

\begin{abstract}
In UMTS network, radio planning cannot only be based on signal predictions, but it must also consider the traffic distribution, the power control mechanism as well as the power limits and the signal quality constraints. The present work aims to optimize the number of base stations used in the WCDMA radio network. In this paper, we propose a mathematical programming model for optimizing the base station locations considered for the uplink (mobile to BS) direction, and which considers the typical power control mechanism of WCDMA. The two contrasting objectives of the optimization process are the traffic coverage maximization and the installation costs minimization. A mimetic algorithm (AM) (genetic algorithm+ a local search) is proposed to find good approximate solutions of this NP hard problem. The Numerical results are obtained for reels instances, and generated by using classical propagation models.
\end{abstract}

Keywords-Ukumura-Hata model; UMTS; W-CDMA; Genetic Algorithms.

\section{INTRODUCTION}

Choosing the optimal location sites for WCDMA Network is a big challenge for a telecommunication vendor. Indeed, the deployment of a UMTS network represents a huge investment mainly related to infrastructure costs. In this context, an accurate and efficient optimization of radio access networks become essential to saving these investments, reduce the number of site deployed and to ensure a good quality of service to users [2].

Traditional models of planning adopted in TDMA cellular systems are based on the prediction of signal level and they do not take account of the effect of traffic on the capacity of a cell, or the impact of the control mechanism power on the overall interference level in the system. For UMTS, and due to technology CDMA access [6], the design takes into account the interference and tries to guarantee a certain quality target for each radio link. Although, the capacity will depend on the tolerable level of interference in the system because of strong correlation between coverage and capacity aspects, the choice of site locations must necessarily take into account both these aspects.
Modeling optimization problems are a key step before defining the appropriate methods for their resolution. The problem we deal with is to look for sites to install from a number of potential sites while starting propagation models and well-defined Traffic [1], [3]. The choice of sites WCDMA positions can be formulated mathematically as an optimization problem under constraints, an objective function that involves financial costs and other costs related to the amount of power transmitted in the system.

\section{LOCATION MODEL FOR UPLINK DiRECTION}

Consider a territory to be covered by UMTS service. A is a set of candidate sites, $A=\{1, \ldots, s\}$ where the base stations can be installed and $\mathrm{T}$ a set of test point (TP), $\mathrm{T}=\{1, \ldots, \mathrm{m}\}$. TP is a relatively small region of the service area from which one or more connections can be initiated.

Each site $\mathrm{j}$ has an installation cost noted $\mathrm{Cj}$. We denote by ni the number of simultaneous connections by TP. Thus, it can match the number of simultaneous active connections or by TP. This is a feature that can be refunded after the study and analysis of traffic requested in the study area. Information propagation are also known. Indeed, let $F=\{$ fij $\} 1$ $<=\mathrm{i}<=\mathrm{m}, 1<=\mathrm{j}<=\mathrm{s}$ is the propagation gain matrix, where fij $0<\mathrm{fij}<1$, is the propagation factor [3], the radio link between a $\mathrm{TP} \mathrm{i}$ and $\mathrm{j}$ a potential site.

In the problem of locating sites WCDMA we wish to select a subset of the set A of potential sites where base stations will be installed, and assign the TPs suitable sites taking into account the requested traffic, quality of service terms of SIR and installation costs.

The problem of the location of base stations in the context of UMTS in the direction uplink is modeled by the following optimization problem:

$$
\min \Sigma_{\mathrm{j}=1}^{\mathrm{s}} \mathrm{C}_{\mathrm{j}} \mathrm{a}_{\mathrm{j}}+\mu \Sigma_{\mathrm{i}=1}^{\mathrm{m}} \Sigma_{\mathrm{j}=1}^{\mathrm{s}} \mathrm{n}_{\mathrm{i}}\left(1 / \mathrm{f}_{\mathrm{ij}}\right) \mathrm{b}_{\mathrm{ij}}
$$

Under the constraints:

$$
\sum_{\mathrm{i}=1}^{\mathrm{m}} \mathrm{b}_{\mathrm{ij}}=1 \quad \text { i } \varepsilon \mathrm{T}
$$




$$
\begin{array}{ll}
\mathrm{b}_{\mathrm{ij}}<=\mathrm{a}_{\mathrm{j}} & \mathrm{i} \varepsilon \mathrm{T} ; \mathrm{j} \varepsilon \mathrm{A} \\
\mathrm{b}_{\mathrm{ij}}, \mathrm{a}_{\mathrm{j}} \varepsilon\{0,1\} & \mathrm{i} \varepsilon \mathrm{T} ; \mathrm{j} \varepsilon \mathrm{A}
\end{array}
$$

The first term of the objective function is the sum of the cost of installation sites. Thus, the ratio 1 / fij is proportional to the power emitted by a TP $\mathrm{i}$ assigned to the BS (Base Station) j, the second term corresponds to the total power emitted. $\mu>=0$ is the parameter difference between the two objective functions. Constraint (2) ensures that TP can be connected to a single base station. Constraint (3) requires that the TPs are assigned only to sites where base stations are installed. Both decision variables, bij, aj, have binary values 0 or 1 .

In UMTS, the mobile is much more limited in power than GSM network [6]. The constraint of limited power states that TP cannot be connected to a site with a power greater than it can deliver. Another fundamental aspect is the quality of the signal received by each BS that identifies the ability of a site [4]. It defines the number of connections supported by a base station (BS). The criterion adopted for a TP to communicate with such a base station is based on the value of the SIR. Indeed, a TP is connected to a particular site if the value of the SIR is greater than or equal to a minimum value. For each connection it embodies this constraint by the following inequality [3]:

$$
\mathrm{SIR}=\mathrm{SF} * \mathrm{P}_{\text {reçue }} / \mathrm{I}_{\text {intra }}(1+\mathrm{f})>=\mathrm{SIR}_{\text {min }}
$$

We assume that the interference caused by neighboring cells $\left(\mathrm{I}_{\text {extra }}\right)$ can be expressed by $\mathrm{f}$ factor interference caused by other connections of the same cell $\left(\mathrm{I}_{\text {intra }}\right)$. Received power $\mathrm{P}_{\text {received }}$ by a base station $\mathrm{j}$, for a TP assigned to this site, is equal to a power $\mathrm{P}_{\text {target }}$. Our final model simplifies the problem of locating sites which is among NP-hard problems [1], [2], [7].

For the model more realistic, the interference must be explicitly extracellular and intracellular interference considered independently. However, for an uplink connection between TP $\mathrm{i}$ and base station $\mathrm{j}$, there is no significant difference between the two types of interference [6]. With a received power based power control mechanism, the overall interference in the system for each potential site $\mathrm{j} \varepsilon \mathrm{A}$, has the following expression:

$\mathrm{P}_{\text {target }} /\left(\Sigma_{\mathrm{h}=1}^{\mathrm{m}} \mathrm{n}_{\mathrm{h}} \mathrm{f}_{\mathrm{hj}} \Sigma_{\mathrm{t}=1}^{\mathrm{s}}\left(\mathrm{P}_{\text {target }} / \mathrm{f}_{\mathrm{ht}}\right) \mathrm{b}_{\mathrm{ht}}-\mathrm{P}_{\text {target }}\right)>=\operatorname{SIR}_{\min } \mathrm{a}_{\mathrm{j}}$

Where the thermal noise is neglected. This constraint assumes that if a base station is installed at the site $\mathrm{j}$ (ie, $\mathrm{j}=1$ ), the value of the SIR resulting exceed the given value of $\operatorname{SIR}_{\min }$.

\section{OptimizAtion Methods AND SOME COMPUTATIONAL RESULTS}

\section{A. Basic principle of genetic algorithm}

The problem of locating base stations of a UMTS network is an NP-hard problem [1-2]. To solve this problem we propose an algorithm memetic (AM) based on genetic algorithms methodology and local search.

Genetic algorithms rely heavily on a binary encoding string $0 / 1$ fixed length $s * m$ represents a configuration (the problem) solution called individual. Genetic operators: crossover, mutation and selection [7], are defined to operate on a random person or two to generate new configurations. GAs are generally sensitive to the distribution of the initial population. The adaptation of genetic operators in our problem and hybridization with local search makes our method can produce good quality results. Indeed, the two genetic operators allow the AM to exploit and explore the search space effectively guiding the search towards the most promising regions.

Rather than deterministically decide in favor of individuals best adapted, GAs do that by fostering probabilistic rules. This leaves little room for those less well to survive and reproduce. In order to better promote this principle, we have introduced the local search in each iteration of the genetic algorithm (Figure 1) with a probability pls.

The step of evaluating the fitness function allows selection of the best individuals created, by ordering the best fitness of the worst individual in the population size of 100. The search stops when the number of iterations limit is reached.

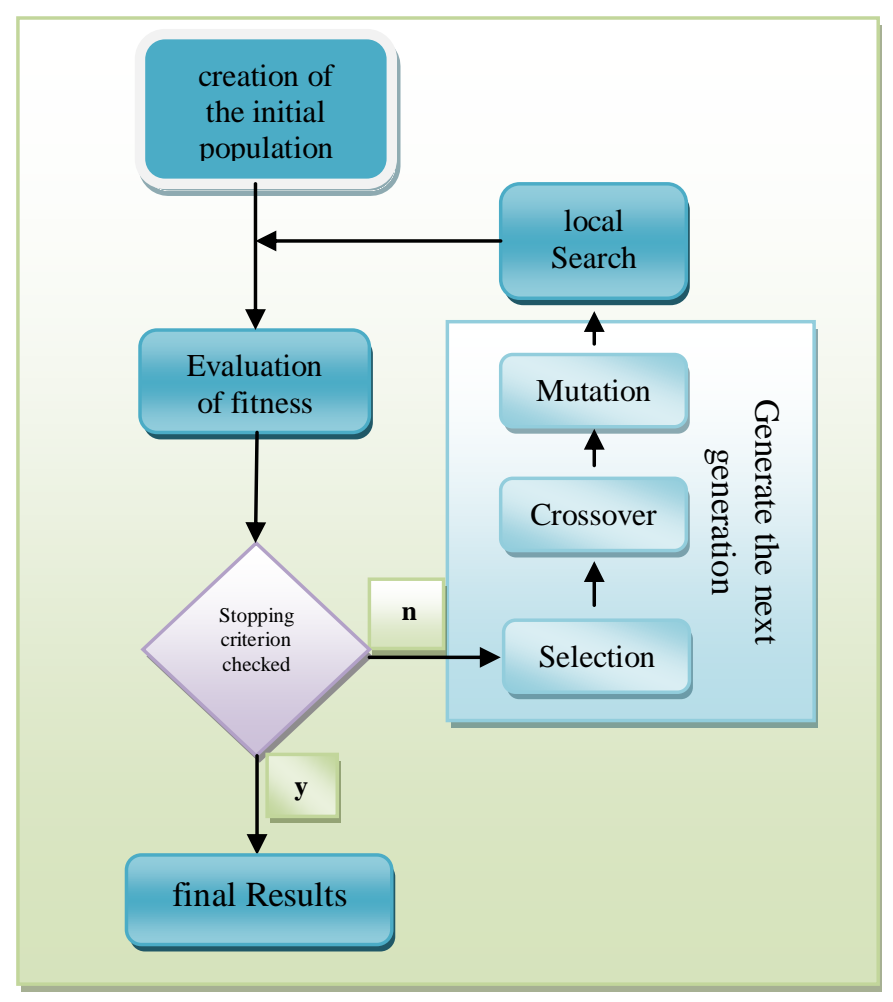

Fig.1. Optimization model using genetic algorithm and local search

\section{B. Numerical results}

In our simulation, we will focus on the basic model. The power control is important for the proper functioning of a WCDMA network. Indeed, it can meet the needs of users and minimize the overall interference in the network. For this, we assume that the power control mechanism is perfect and is based on the received power.

We studied the case of an urban environment and multiservice, voice service speed $12.2 \mathrm{kbps}$ data service rate $64 \mathrm{kbps}$ and 384 kbps. We model Okumara-Hata [3] as a model of propagation attenuation is given by the equation:

$A_{u}=69,55+26,16 \log (F)-13,82 \log \left(H_{b}\right)-\left[(1,1 \log (F)-0,7) H_{m}\right.$ $-(1,56 \log (\mathrm{F})-0,8)]+\left[44,9-6,55 \log \left(\mathrm{H}_{\mathrm{b}}\right)\right] \log (\mathrm{d})$ 
Where $\mathrm{F}$ is the frequency signal in megahertz, $\mathrm{H}_{b}$ and $\mathrm{H}_{\mathrm{m}}$ are respectively the height of the base station and the height of the mobile in meters, $\mathrm{d}$ is the distance in kilometers.

For the simulation, we consider the following parameters shown in Table 1 [1]:

TABLE I. SIMULATION PARAMETERS

\begin{tabular}{|l|l|}
\hline \multicolumn{1}{|c|}{ Parameters } & \multicolumn{1}{|c|}{ values } \\
\hline frequency (en Mhz) & 2000 \\
\hline height of TPs (in meters) & 2 \\
\hline Height sites (in meters) & 10 \\
\hline Power target TPs (in dBm) & -100 \\
\hline Maximum power of TPs (in dBm) & 30 \\
\hline SIR $_{\text {target }}$ for voice service (in dB) & 6 \\
\hline SIR $_{\text {target }}$ for data service $64 \mathrm{kbps}($ in $\mathrm{dB})$ & 3.2 \\
\hline SIR $_{\text {target }}$ for data service $384 \mathrm{kbps}$ (in dB) & 1 \\
\hline Number of connections by TP & 1 \\
\hline
\end{tabular}

Distribution by a uniform law to location of TPs. The instances with medium-size are characterized by service area size of $400 * 400 \mathrm{~m}, \mathrm{~m}=95$ TPs and $\mathrm{s}=22$ potential sites. The cost of installation sites is constant $\mathrm{Cj}=\mathrm{C}$ for all $\mathrm{j}$ of $\mathrm{A}$. Minimum activated sites (Figure 2) among 22 potential sites are between 7 and 9 sites for 4 distributions ZU1 to ZU4 (urban area). Each ZUi is executed 20 times (Table 2). The required traffic is always satisfied.

The dissimilarity of the number of connections in each site installed by our metaheuristic algorithm, due to the random distribution of the potential number of TPs in the area to be covered. The aspect of the quality of the signal received by each BS for identifying the ability of a site defines the number of connections supported by a given base station. The criterion adopted for a TP to communicate with such a base station is based on the value of the SIR, the results obtained with the SIR model for the uplink case with real instances generated and granted propagation model. Noting that in uplink, the number of sites installed is much more important than in downlink. Indeed, in downlink intra interference is less significant than in uplink because each BS uses these orthogonality codes and the entire couvrement is still obtained with a minimum number of BSs. Otherwise, in downlink direction we consider a diversity of variable power received by the TPs, whereas in uplink it is sufficient to consider a single received power for each BS.

\section{CONCLUSION}

We studied a model to optimize the location of base stations in the UMTS standard and in the uplink case. Indeed, the hybridization of genetic algorithm and local search has allowed us to install an optimal number of base stations while satisfying the constraints of quality of service, power, capacity and total network coverage on the zone concerned.
TABLE II. RESULTS OBTAINED FOR $\mathrm{m}=95, \mathrm{~s}=22$

\begin{tabular}{cccc}
\hline area & \# of BSs & $\begin{array}{c}\text { Execution time } \\
(\mathbf{m n})\end{array}$ & \# average \\
\hline ZU-1 & 8 & 13 & 8.5 \\
\hline ZU-2 & 7 & 12 & 7.4 \\
\hline Z-U3 & 8 & 12 & 8.4 \\
\hline ZU-4 & 8 & 13 & 8.6
\end{tabular}

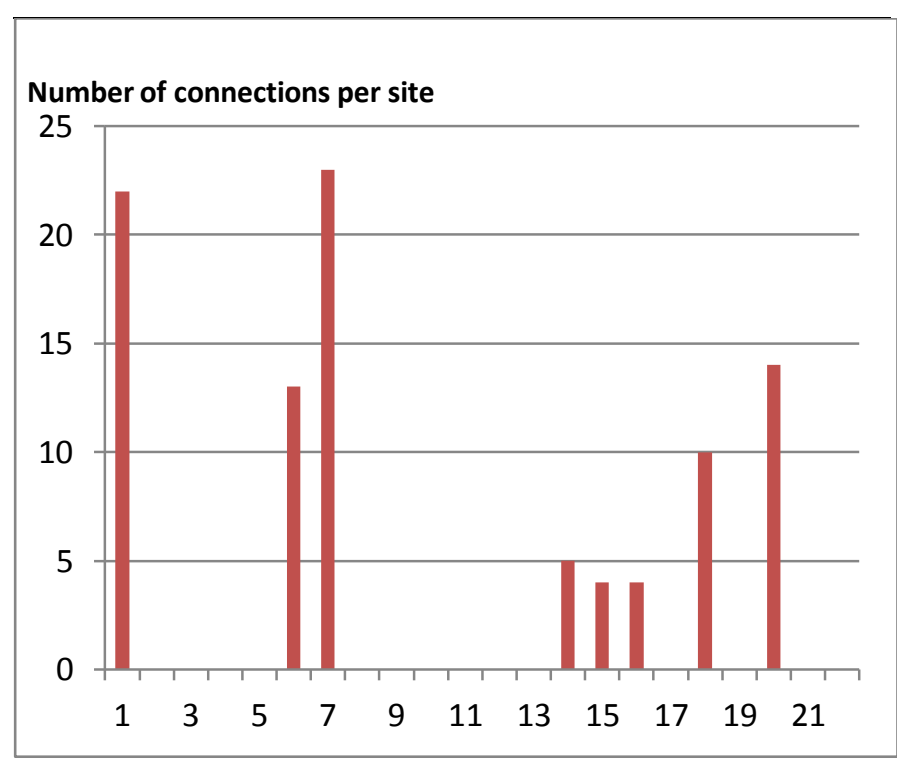

Fig.2. number of TP connected to each site $\mathrm{j}$

The memetic algorithm has shown high efficiency in order to don't have a premature convergence and to avoid local optima. Our study focused on voice and data services with a classical propagation model. As perspective to this work, we aim to run simulations of larger instances, and study the same mathematical model with other algorithms.

\section{References}

[1] E. Amaldi, A. Capone, F. Malucelli. Optimization models and algorithms for downlink UMTS radio planning. Manuscript, (2003).

[2] E. Amaldi, A. Capone, F. Malucelli. Optimizing base station siting in umts networks. In Proceedings of IEEE VTC Spring (2001).

[3] M. Hata. Empirical formula for propagation loss in land mobile radio service. IEEE Trans. On Vehicular Technology, (29:317-325, 1980).

[4] R. Mathar and T. Niessen. Optimum positioning of base stations for cellular radio networks. Wirelless Networks, pages $(421-428,2000)$.

[5] C. Oliva. Techniques hybrides de propagation de contraintes et de programmation mathématique. Thèse, Université d'Avignon et des Pays de Vaucluse, (2003).

[6] J. Laiho, A. Wacker, T. Novasad. Radio network planning and optimization for UMTS. Copyright by John Wiley and Sons, (2002).

[7] J.Dréo, A.Pétrowski, P.Sinrry, E.Taillard. Metaheuristics for Hard Optimization, Springer (2006). 